\title{
Gains in Life Expectancy by Elimination of Specified Causes of Death in Pakistan
}

\author{
Syed Mubashir Ali and Zafar Mueen Nasir*
}

\section{INTRODUCTION}

Death is inevitable. However, efforts have always been made to delay it. Due to the advancement in medical science, the developed countries have succeeded in achieving a considerable increase in the life expectancy of the people. On the other hand, the developing countries are striving hard to follow the same trend, but with lesser success.

Constrained by limited resources, health planners have been compelled to set priorities towards the elimination of widespread fatal diseases. The cause specific death rates can provide a measure of the most widely prevalent diseases in the region. An age and cause specific death rate, would give a more refined measure of the same. However, in order to measure the gain in life expectancy by elimination of specific causes of death, the use of the life table technique would be an appropriate one. For example, if 'Malaria' is the largest killer in a region, the application of this technique could provide us with added years of life resulting from the elimination of Malaria. The present study is an attempt to examine the gains in life expectancy at birth as well as for other broad age groups by eliminating specified causes of death.

\section{DATA AND THEIR LIMIT ATION}

The data for the present analysis have been taken from the 1984 Pakistan Demographic Survey (PDS) report (Government of Pakistan, 1987). The survey was carried out by the Federal Bureau of Statistics (FBS) at the national level covering 21965 households comprising of 141849 household members. The age and

*The authors are Research Demographer and Staff Economist respectively, at the Pakistan Institute of Development Economics. They are grateful to Mr Mohammad Afzal, Chief of Research (Demography) for his constant encouragement and guidance during the preparation of this paper. They are indebted to Dr Mohammad Irfan, Chief of Research (Economics) for his useful suggestions and comments. Their thanks and gratitude are due to Dr A. S. Nasir, a retired WHO Advisor for his help and guidance in identifying various groups of diseases according to their etiological similarity. This paper is an abridged version of the one read at the Fifth Annual General Meeting of Pakistan Society of Development Economists. 
cause-wise mortality data at national level in this survey, is a pioneer attempt. The absence of any other comparable data makes it difficult to comment on the credibility of this data. Inaccurate diagnosis of the disease as cause of death may occur as Padley (1959) noted in Sri Lanka where prolonged fever could be diagnosed wrongly as typhoid or pyrexia of unknown origin. Yet our data seem to be fairly reliable as the proportion of ill-defined and unknown causes of death is considerably low.

As pointed out by Zaki and Zaki (1981), the quality of reporting, particularly that of age, has been found to be inaccurate in Pakistan. But despite this fact, we maintain the originality of the data and do not apply a graduating or smoothing technique. This is because our purpose is to demonstrate the respective gain in life expectancy by elimination of various causes of death rather than to determine the levels of life expectancy.

It would have been all the more appropriate to estimate gains in life expectancy sex-wise as many causes of death are closely correlated to it. We however, restrict our analysis to both sexes combined as data breakdown by sex are not available. A study based on the 1971 Population Growth Survey (PGS) indicates urbanrural differentials in cause specific death rates in Pakistan (Irfan, 1986). Nonavailabilty of the data by urban and rural breakdown do not permit us to carry out such an analysis here.' The list of 50 causes of death as reported in the 1984 PDS are classified into 11 broad groups according to their etiological similarity.

\section{METHODOLOGY}

The methodology for the construction of 'regular life table' (all causes combined) and 'cause of death deleted tables' have been adopted from Preston et al. (1972). The $n M x$ values were provided as input. ${ }^{2}$ The $n M x$ values for 'regular life table' and 'cause of death deleted tables' were estimated by the following formula:

$$
n M x=\operatorname{ASMR}(i)=\frac{D i}{P i} \text { for (all causes combined) }
$$

and $n M x(c)=\operatorname{ASMR}(i, C j)=\frac{D(i, C j)}{P(i)}$ for (causes of death deleted tables)

where $\operatorname{ASMR}(i)=$ Age-specific Mortality Rate of $i$ th age group

$$
D i=\text { Number of deaths of } i \cdot \text { th age group }
$$

'We hope to further extend this analysis sex-wise and for urban-rural areas as soon as the PDS data tape is available to PIDE.

${ }^{2}$ In the analysis, iqo (probability of death at age less than one year) has been replaced by Infant Mortality Rate (IMR)

\section{$P i=$ Population of $i$ th age group.}

and $\operatorname{ASMR}(i, C j)=$ Age-specific Mortality rate of $i$ th age group when $j$ th cause of death is eliminated from causes of death.

$D(i, C j)=$ Number of deaths of $i$ th age group when $j$ th cause of death is eliminated from causes of death.

\section{$P(i)=$ Population of $i$ th age group.}

For the age groups under one year and 1-4 years, Coale and Demeny (1966) separation factors for South Model Life Tables were used. ${ }^{3}$ The separation factors for all higher age groups is taken as 2.5 .

To estimate the gains in life expectancies by eliminating each group of deathcausing diseases we have constructed in all twelve life tables - one for all causes combined and others after eliminating specific groups of death-causing diseases. The 'cause of death deleted tables' have been constructed on the assumption that causes of death are mutually exclusive. The gains in the expectation of life resulting due to the elimination of specific groups of death-causing diseases will be estimated by the difference in life expectancies between 'regular life table' (all causes combined) and 'cause of death deleted tables'. In mathematical notation:

$$
\Delta E(i)=E(i, C j)-E(i)
$$

where $\quad E(i)=$ Life expectancy for $i$ th age group given all the causes of death.

$E(i, C j)=$ Life expectancy for $i$ th age group when $j$ th cause of death is deleted from causes of death.

In order to measure the impact of specified death-causing diseases on the working age population, we have to estimate the gains in the expectancies of life of population in the productive years that is, population between 10-64 years. The added years of life for this population have been estimated by the difference of the quantity $(\mathrm{T} 10-\mathrm{T} 60) / \ell 10$ in the regular life table and cause of death deleted tables, where $T x$ is the person years lived by hypothetical life table cohort above age $x$ and $\ell 10$ is the number of survivors to age 10 in hypothetical life table cohort.

\section{RESULTS}

Table 1 shows the gain in life expectancies at birth and at working ages, after

${ }^{3}$ In an exercise of comparing survival ratios, the survival ratios of South Model Life Tables were found to be closest to our ratios. 
eliminating specific causes of death in Pakistan. According to this table, the maximum number ( 3.88 years) would be added to the life expectancy at birth if 'Malaria' were eliminated as a cause of death. This is followed by 2.72 years by elimination of 'Tuberculosis' all forms, 2.35 years by 'children diseases', 1.99 years after eliminating 'Miscellaneous diseases'. Elimination of 'water-borne diseases' would add 1.91 years. Elimination of 'other infective and parasitic diseases' and the diseases of the 'circulatory system' would add 1.83 and 1.29 years respectively.

Accidents particularly those caused by automobilies, have ostensibly resulted in a large number of deaths, yet our data do not indicate so: Only 0.84 years of life would be added to the life expectancy at birth if there were no deaths due to accidents. Neoplasm is another fatal disease presumed to be considerably prevalent in Pakistan. But elimination of this disease also shows a gain of only 0.42 years in life expectancy.

The nat ure of widespread diseases found in developed regions are to different those found in underdeveloped regions. For example, in the United States, the greatest gain (13 years for males and 17 years for females in 1964) in life expectancy would result from the elimination of 'circulatory system' diseases (Preston et al., 1972). The same pattern was observed in most of the developed countries where large gains (in years) in life expectancy could be due to the elimination of the same disease. In contrast, the elimination of 'circulatory system' diseases in Pakistan would show a gain of only 1.29 years (Table 1 ).

In most of the developed world, the prevalence of infective and parasitic diseases' is so low that elimination of these contributes to a negligible gain in life expectancy. For example, in the United States only 0.1 year would be added to the life of both males and females if 'infective and parasitic diseases' were eliminated. (Preston et al. 1972). But in Pakistan this group which includes Malaria and Tuberculosis as well, would result in the greatest gain in life expectancy, if eliminated. Another study based on 165 populations showed a marked difference in added years of life accrued to the elimination of certain diseases such as infective and parasitic diseases, Neoplasm, Cardio-Vascular diseases and Diarrhoael diseases between high and low mortality populations (Preston 1976).

As already mentioned, the group of 'Miscellaneous diseases' if eliminated would bring about a considerable gain in life expectancy. This group includes the largest number of diseases; each one of them not very important by itself but collectively making a significant gain. Only 'Allergic disorders' was relatively conspicuous causing roughly 30 percent of the total deaths in this group.

The working age group is scarcely influenced by most of these diseases. The cardio-vascular diseases are perceived to be considerably fatal between 40-50 years of age in developing countries. A study based on hospital data in Pakistan also depicts the same trend (Irfan 1986). However our data do not show much change in life 
expectancy of the working population, that is 10-64 years by eliminating these diseases, including cardio-vascular diseases. As a matter of fact, this pattern of mortality is in accordance with a $U$-shaped mortality curve, typical of a high mortality population, where death rates are high at infancy and early childhood, drop to a minimum at about ages $10-14$, remaining more or less flat at ages $20-24$, and then rising gradually to a maximum at very advanced ages.

\section{CONCLUSION}

Pakistan presents a mortality pattern typical of a high mortality population where children's diseases, infective and parasitic and water-borne diseases are widespread. Elimination of these could raise the average length of life considerably. In developed countries, the health planners, by adopting preventive health measures against these diseases and by providing an overall improved environment were able to control these diseases to a considerable extent. Lately, the health planners in Pakistan seem to have realised the importance of preventive measures. The vaccination campaign against infants and children's diseases has been introduced countrywide. However, a lot more remains to be done for the containing of infective and parasitic diseases including Malaria and Tuberculosis.

Diffusion of proper health education highlighting the risk of smoking and intake of proper diet can reduce the risk of death due to diseases of the circulatory system - another leading cause of death in Pakistan. Moreover, an overall improvement in the environment by increasing the supply of potable drinking water, better sewerage and other sanitary facilities would provide many added years of life to the population at large.

\section{REFERENCES}

Coale, A. J. and Paul Demeny (1966). Regional Model Life Tables and Stable Population. New Jersey: Princeton University.

Irfan, Mohammad (1986). Mortality Trends and Patterns in Pakistan. Bangkok: ESCAP. (Asian Population Studies Series No. 75)

Padley, R. (1959). Causes of Death Statements in Ceylon: A Study in Levels of Diagnostic Reporting. Bulletin of World Health Organization.

Pakistan, Government of (1987). Pakistan Demographic Survey. Karachi: Federal Bureau of Statistics.

Preston, H. Samuel (1976). Mortality Patterns in National Population with Special Reference to Recorded Causes of Death. New York: Academic Press.

Preston, H. Samuel, Nathan Keyfitz and Robert Schoen (1972). Causes of Death: Life Tables for National Population. New York: Seminar Press.
Zaki, K. P., and M. J. A. Zaki (1981). A Comparative Study of Age Reporting in Selected Censuses and Surveys in Pakistan. Islamabad: Pakistan Institute of Development Economics. (Research Reports Series No. 130) 


\section{Comments on}

"Gains in Life Expectancy by Elimination of Specified Causes of Death in Pakistan"

This is the first ever attempt to construct life tables based on causes of deaths. Life Tables are constructed by eliminating deaths by specified causes and showing gains in life expectancies due to these eliminations. The comments on the contents of the paper are given as under:

(1) The data for the paper has been taken from the 1984 Pakistan Demographic Survey. The 1984 PDS Report does not give the required data by age and cause of death. However, if the authors have been successful in getting the relevant data, then it is not clear as to whether this is based on weighted figures or sample figures. In case sample figures are used, then it is doubtful that the results of the study would yield the real picture of the situation. As far as the sample weights are concerned, in the current case these vary by a very wide margin and hence, the aggregation of sample figures carries no meaning.

(2) Morbidity, mortality and population statistics are always subject to age misreporting errors. Any study which is based on ages should first examine the age misreporting pattern and then should try to adjust the data accordingly, if possible.

(3) Morbidity and mortality data are always age-sex dependent, and they vary between sexes in urban, rural areas and also across the regions. This study is based solely on the combined data of the sexes and as such is not likely to yield a true picture of the variability of expectation of life by sexes. Morbidity and mortality vary differently right from infancy till old age for each sex.

(4) In developing countries, deaths are underenumerated to a much greater extent as compared to births. Dr Irfan has shown in his paper that deaths in Pakistan are enumerated to the extent of 70 percent. This point needs to be taken care of in this study, and some part of adjustment factor should be developed to correct the mortality data.

(5) The authors have produced eleven life tables by eliminating eleven causes. It would have been appropriate if fewer life tables were developed through regrouping the causes of death. The first three causes seem to be more appropriate in the present case but if the data becomes available by sex as well as by rural/urban areas, then the study could have been a major breakthrough in the study of mortality in Pakistan.

The authors deserve congratulations for really bringing to light a topic which has not been touched upon as yet by the social scientists. Studies on mortality need further research as this subject has been really neglected in the past mainly because the major aim of the government has been to influence a decline in fertility. It is however assumed that mortality continues to decline just because of the impact of health and other socio-economic development programmes.

M. Naseem Iqbal Farooqui

NIPS,

Islamabad 